\title{
19 University
}

Maciąg, J. et al. (2017) Systemic T cells and monocyte characteristics in patients with denture stomatitis. Journal of Prosthodontics, 26(1), pp. 1928. (doi:10.1111/jopr.12447)

This is the author's final accepted version.

There may be differences between this version and the published version. You are advised to consult the publisher's version if you wish to cite from it.

http://eprints.gla.ac.uk/134413/

Deposited on: 17 January 2016

Enlighten - Research publications by members of the University of Glasgow http://eprints.gla.ac.uk 


\section{Systemic T cells and monocyte characteristics in patients with denture stomatitis.}

Joanna Maciąg ${ }^{1}$, Tomasz Mikołajczyk ${ }^{2}$, Paweł Matusik², Ryszard Nosalski², Agnieszka Sagan ${ }^{2,4}$, Anna Maciąg ${ }^{3}$, Daniel Nowakowski ${ }^{1}$, Grzegorz Wilk², Grzegorz Osmenda², Tomasz Guzik², Marta Cześnikiewicz-Guzik ${ }^{1,5}$

${ }^{1}$ Institute of Dentistry, Department of Dental Prophylaxis and Experimental Dentistry, Jagiellonian University Medical College, Cracow, Poland;

${ }^{2}$ Department of Internal and Agricultural Medicine, Jagiellonian University Medical College, Cracow, Poland;

${ }^{3}$ Zbigniew Żak Voivodeship Dental Clinic, Cracow, Poland;

${ }^{4}$ Institute of Cardiovascular and Medical Sciences, University of Glasgow, Glasgow, UK;

${ }^{5}$ Oral Sciences Research Group, Dental School, University of Glasgow, Glasgow, UK

Running title: Denture stomatitis and peripheral immune system.

Address for Correspondence:

Marta Czesnikiewicz-Guzik

Oral Sciences Research Group, Glasgow Dental School, School of Medicine, College of Medical, Veterinary and Life Sciences, University of Glasgow

378 Sauchiehall Street, room F15

Glasgow G2 3JZ

Email: marta.czesnikiewicz-guzik@glasgow.ac.uk

Phone: 004407557674687

Financial support: Public Funds for Science of Republic of Poland grant Nr 2997/B/P01/2009/36 and the Foundation for Polish Science Welcome Grant (FNP/Welcome/02); Cracow; Poland and statutory grant K/ZDS/003967 


\section{Abstract}

Purpose: Chronic inflammatory disorders of the oral cavity, such as periodontitis were recently linked to the systemic immune activation. Since fungal oral infections were not studied in this respect yet, the aim of our study was to determine whether the local inflammation caused by oral fungal infection of the palatal tissue (denture stomatitis - DS), is associated with the systemic inflammatory response. This problem is becoming essential in relation to ageing of population.

Materials and Methods: Peripheral blood of DS patients $(n=20)$ and control patients $(n=24)$ was assessed with flow cytometry to determine lymphocytes and monocytes profile. Intracellular cytometric analysis was carried out to establish cytokine production by T cells. DS was diagnosed based on clinical symptoms of DS such as swelling and redness of oral mucosa, confirmed by microbiological swabs for fungal colonization with Candida species. Control group was recruited from complete-denture users without clinical and microbiological signs of oral infections.

Results: Percentages of peripheral lymphocytes, T cells, monocytes and their subpopulations were similar in both studied groups. The exception was median percentages of CD25+ T cell subsets, which were significantly lower in DS patients than in control subjects. This reduction was observed in both CD4 T cell subset $(16.7 \%$ and $28.1 \% ; p=0.0006)$ and CD8 T cells $(4.6 \%$ and $7.0 \% ; p=0.007)$

Conclusions: While DS and associated local fungal infection does not overtly affect activation of monocytes or lymphocytes, the number of CD $25+T$ cells is significantly lower in the DS patients, possibly indicating limited potential for the infection clearance in denture using ageing subjects.

KEYWORDS: systemic inflammation, Candida albicans, denture stomatitis, lymphocytes, monocytes, immune system

CCR5 - C-C chemokine receptor type 5

$C D$ - cluster of differentiation

DS - denture-related stomatitis

FSC - forward-scattered light channel

HLA - human leukocyte antigens

IFN - interferon

IL - interleukin

PBMC - peripheral blood mononuclear cells

RANTES - Regulated on Activation, Normal T-cell Expressed and Secreted chemokine

SD - standard deviation 
SSC - side-scattered light channel

Th - T helper cells

TNF - Tumour Necrosis Factor

Treg - regulatory T cells

Q1 and Q3 - 25th (Q1) and 75th (Q3) percentiles 


\section{Introduction}

Denture stomatitis is an important clinical problem affecting quality of patients' life and, as recently indicated, may co-incide with selected systemic co-morbidities including vascular dysfunction ${ }^{1}$. The mechanisms of these co-morbidities are unclear, mainly because it is not clear to which extent immunopathogenic process in the oral cavity of patients with denture stomatitis affects systemic immunity.

Recent years have brought better understanding of the importance of chronic, low-grade inflammation in mediating the risk of cardiovascular diseases, cancer as well as rheumatoid disorders. Oral cavity, which is inhabited by 500 different species of microorganisms forming biofilm, is a very important potential site of initiation and propagation of such low-grade inflammation. While numerous studies have focused on the role of periodontitis or periapical changes as sites of origin of low grade inflammation ${ }^{2}$, recent studies have suggested that denture stomatitis might also represent such chronic low grade inflammation.

The systemic characteristics of $T$ cells or monocytes in denture stomatitis (DS) have not been described. DS is a fungal infection, most commonly with Candida species. They are part of commensal flora of the oral cavity, however the combination of denture presence together with factors such as: low salivary $\mathrm{pH}$, high sugar consumption ${ }^{3}$ or low saliva flow contribute to excessive fungal growth. Mucosal immune response to fungal pathogens include neutrophils as major effectors of the innate response, driven by cytokines released from epithelial cells after contact with pathogen ${ }^{4,5}$. However, epithelial cells produce also $\beta$-defensin 2 and CCL20 orchestrating dendritic cell recruitment ${ }^{6}$ which present fungal antigens to the T cell. It was long thought, that Th1 are the leading type of response for fungal infection, but after discovering the existence of IL-17 producing Th17 cells, this type of immune reaction appears to be vital ${ }^{7}$, with suppressive $T$ regulatory (Treg) cells playing role in counter-balancing the Th17 response ${ }^{6}$. Mice lacking IL-17 signalling (IL-23\% or IL$17 \mathrm{~A}$ receptor $^{-1}$ ) are more susceptible for oral candidiasis than wild-type mice ${ }^{8}$. Similarly, in humans genetic variations of IL-17 related genes are associated with increased susceptibility ${ }^{9-12}$, to chronic mucocutaneous candidiasis. It remains unclear, how are these local immune responses reflected by the changes in systemic immunity and subpopulations of proinflammatory T cells and monocytes in peripheral blood of DS patients. There are limited studies suggesting that oral candidal infection may impact peripheral blood mononuclear cells and cytokine production in vitro in response to Candida antigens $^{13,14}$ is altered, although it is not clear which type of the cells: monocytes or lymphocytes primarily response.

Therefore, the aim of our study was to determine whether the local inflammation caused by presence of denture-related stomatitis, coincides with the systemic inflammatory response, depicted as activation of peripheral blood immune cells

\section{Methods}

Patients and clinical evaluation: Consecutive patients using dental prostheses for at least 6 months were recruited to the study and assigned to two groups: DS patients $(n=20)$, and control non-DS $(n=$ 24) subjects, who presented with healthy oral mucosa ${ }^{1}$. 
Detailed methodology, clinical data acquisition and exclusion criteria along with major systemic clinical features were described previously ${ }^{1}$. In brief, patients' oral mucosa was examined to detect the presence of clinical symptoms of DS such as: erythema, swelling. Additionally, patients' subjective complains, such as such as discomfort and pain in the palatal area were registered. The swabs from the inflamed area have been taken for the routine microbiological laboratory diagnostic tests for Candida species presence to confirm clinical diagnosis. Swabs were taken from the hard palate (between the second and third palatal fold). Samples were collected after an overnight fast and after at least 6 hours of continuous denture usage, without the use of adhesives or rinsing the mouth with disinfectants. Samples were collected by gently rubbing a sterile cotton swab moist with saline over the palatal tissue and then subsequently inoculating a primary isolation in dextrose agar transport medium. The collected sample was transported to the laboratory immediately after patients' visit. Candida identification was made according to the colour of the colony on CHROMagar Candida medium (Graso Biotech, Starogard Gdański, Poland) after 36 to 48 hours of incubation in $36^{\circ} \mathrm{C}$. Patients were recruited to DRS group when clinical symptoms were confirmed by positive microbiological culture of Candida species from the palatal tissue. In patients with some teeth present, the periodontal examination was carried out to exclude patients with periodontitis (one or more periodontal pocket depth $\geq 4 \mathrm{~mm}$ ). Exclusion criteria also included: acute inflammatory disorders and chronic inflammation other than DS, neoplastic disease and relapses and chemotherapy courses less than 5 years before the enrolment, antibiotics usage in less than 4 weeks or anti-inflammatory drugs regiment (steroids and non-steroidal, excluding aspirin in doses less than $80 \mathrm{mg}$ ) in less than 2 months before the enrolment. Patients with history of myocardial infarction, acute coronary incident or vascular inflammation in 5 weeks or less before the enrolment, chronic haematological disorders and immunodeficiency or major medication changes during less than 5 weeks before or during study were also excluded.

The study was independently reviewed and approved by local ethics committee of Jagiellonian University, Cracow, Poland. Written informed consent was obtained from all patients and study was conducted in accordance with the Declaration of Helsinki. This study has been registered in ClinicalTrials.gov (identification no. NCT02166450). Information about systemic risk factors of DS such as diabetes (fasting glucose level $\geq 7 \mathrm{mmol} / \mathrm{L}$ or $\mathrm{HbA} 1 \mathrm{c}>6.5 \%$ or current treatment with insulin or oral hypoglycaemic agents), and smoking (current or within last 6 months) were based on patient medical records and detailed patient history. Definition of clinical risk factors was based on European Guidelines on cardiovascular disease prevention in clinical practice from $2012^{15}$.

Extracellular cytometric analysis: Blood samples were collected in ethylenediaminetetraacetic acidcontaining tubes (Becton Dickinson (BD), Franklin Lakes, NJ, USA). PBMC were isolated using Lymphocyte Separation Medium LSM 1077 (PAA Laboratories GmbH, Pasching, Austria). After isolation, cells were washed twice with phosphate-buffered saline with heat inactivated $1 \%$ fetal bovine serum (Gibco, Life Technologies, Carlsbad, CA, USA) and then incubated for 20 minutes at $4^{\circ} \mathrm{C}$ with fluorescently labelled monoclonal antibodies (all antibodies were purchased from BD Pharmingen, San Diego, CA, USA). After washing, cells were re-suspended in phosphate-buffered saline with $1 \%$ fetal bovine serum (Gibco, Life Technologies, Carlsbad, CA, USA) and studied on a FACSCanto II or FACSVerse cytometer (BD Bioscences, San Jose, CA, USA). Results were analyzed using FlowJo vX software (FLOWJO LCC Data Analysis Software, Ashland, OR, USA). Lymphocytes were identified in PBMC on forward-scattered light channel (FSC)/ side-scattered light channel (SSC) scatter plots and T cell presence was confirmed by CD3 staining. Percentages of naive (CD45RA+) and 
memory (CD45RO+) and CD4+ and CD8+ $T$ cells were assessed, as well as presence of surface activation markers in these subpopulations. Monocytes were also identified on FSC/SSC scatter plots with confirmation of CD14/human leukocyte antigens (HLA)-DR staining. Three main populations were assessed among monocytes: CD14highCD16-, CD14dimCD16+ and CD14highCD16+. Both gating strategies are presented on Figures $1 \mathrm{~b}$ and $5 \mathrm{~b}$. The following monoclonal antibodies were used: antiCD3-PerCP (clone SK7), anti-CD45RA/CD45RO/CD3/CD4 BD Multitest ${ }^{\text {TM }}$, anti-CD4-APC (clone RPA-T4), anti-CD4-PeCy7 (clone SK3), anti-CD8-APCH7 (clone SK1), anti-CCR7-PECy7 (clone 3D12), anti-C-C chemokine receptor type 5 (CCR5)-PE (2D7/CCR5), anti-CD69-FITC (clone FN50), anti-CD25-PE (clone MA-251), anti-CD28-APC (clone CD28.2), anti-CD14-APCH7 (clone MøP9), anti-HLA-DR-PeCy7 (clone L243), anti-CD16-PE (clone 3G8). Because of technical reasons, it was impossible to perform monocyte analysis in one patient in DS group.

Intracellular cytometric analysis: Cytokine production by $\mathrm{T}$ cells was assessed after 4-hour incubation of PBMC in Roswell Park Memorial Institute medium 1640 (Gibco, Life Technologies, Carlsbad, CA, USA) with 10\% fetal bovine serum (Gibco, Life Technologies, Carlsbad, CA, USA), gentamycin and L-glutamine (Sigma, Saint Louis, MO, USA) with stimulation with Leukocyte Activation Cocktail with BD GolgiPlug (BD Pharmingen, San Diego, CA, USA). After 20 minutes of on ice staining for cell surface markers (CD3, CD4 and CD8; procedure, suppliers and clones as presented above), cells were washed with phosphate-buffered saline with heat inactivated $1 \%$ fetal bovine serum (Gibco, Life Technologies, Carlsbad, CA, USA). Cells were permeabilized (20 minutes, on ice) (BD Cytofix/Cytoperm, Fixation and Permeabilization Solution, BD Pharmingen, San Diego, CA, USA) and, after washing with BD PermWash Buffer (BD Pharmingen, San Diego, CA, USA), stained for intracellular cytokines for 20 minutes, on ice. Then the cells were washed again with BD PermWash Buffer (BD Pharmingen, San Diego, CA, USA), resuspended in phosphate-buffered saline with $1 \%$ fetal bovine serum (Gibco, Life Technologies, Carlsbad, CA, USA) and studied by flow cytometry, as described for extracellular staining procedure. The following monoclonal antibodies were used: antiIL-17-PE (clone N49-653), anti-IL-4-PE (clone 8D4-8), anti-TNF $\alpha$-FITC (clone MAb11) and anti-IFNYFITC (clone B27). Non-specific staining with isotype-matched control monoclonal antibodies was used (PE Mouse IgG1k Isotype Control, clone MOPC-21 and FITC Mouse IgG1k Isotype Control, clone MOPC-21). All antibodies were from BD Pharmingen, San Diego, CA, USA.

Statistical analysis: Assessment of normality of distribution for all continuous variables was conducted by Shapiro-Wilk test. Variables whose distributions were not consistent with a normal distribution are presented as median and 25th (Q1); 75th (Q3) percentiles and tested by MannWhitney $U$ test. Variables with normal distribution are presented as mean with standard deviation (SD) and tested with Student's $\mathrm{t}$ test. Dichotomous variables were tested by $\mathrm{Chi}^{2}$ test for the expected frequencies equal or higher than 5 . If the expected frequencies were lower than 5, Yates' correction was applied, and Fisher's Exact test was executed for confirmation. Values of $p<0,05$ were considered statistically significant. All analyses were performed with Statsoft Statistica software (StatSoft, Inc., Tulsa, OK, USA).

\section{Results}

Patient characteristics: DS and non-DS groups were comparable in relation to age (mean \pm SD $64 \pm 7$ and $66 \pm 10$, respectively) and gender $(2 \mathrm{M} / 18 \mathrm{~F}$ and $6 \mathrm{M} / 18 \mathrm{~F}$, respectively) and major clinical 
characteristics (Table 1). Compared to the general population, the proportion of females was higher in both groups of subjects using dental prostheses. Proportions of smokers (30\% and $12,5 \%$ ) and diabetic patients (30\% and 8,3\%) were higher in DS group, as these factors are key risk factors for DS, but the difference did not reach statistical significance. Clinical description of DS and control group patients is presented in Table 1. Detailed characteristics describing cardiovascular status of study groups patients was published in the previous article ${ }^{1}$. Most of the screened subjects were edentulous $(n=36)$.

Lymphocyte characterization in peripheral blood of DS patients: Percentages of total lymphocytes and T cells in peripheral blood mononuclear cell (PBMC) of DS and control patients has shown no significant differences (Figure 1a upper panel). Similarly no differences were observed in CD4+ and CD8+ T cell subsets (Figure 1a lower panel). Further studies have shown that presence of DS did not seem to affect median percentages of naive (CD45RA+; 47.15 (31.80; 57.0) and 37.5 (31.55; 49.65); $p=0.25)$ and memory (CD45RO+; $43.15(31.55 ; 55.65)$ and $49.35(40.25 ; 57.40) ; p=0.24)$ T cells. Percentages of the naive (CD45RA+CCR7+), effector (CD45RA-CCR7-) and memory (CD45RA-CCR7+) T cells from both, $C D 4+$ and $C D 8+$ populations, also did not show any differences between the groups (Figure 2a,b).

Lymphocyte activation in peripheral blood of DS patients: Interestingly, we found that the median percentages of cells expressing late activation marker CD25 from CD4+ and CD8+ subsets were significantly lower in the DS group ( $p=0.0006$ and 0.0076 , respectively), but there were no differences between the groups in percentages of CD4+ and CD8+ expressing early activation marker CD69 (Figure 3a). Percentages of CD4+ and CD8+ CD28null subsets were also similar in both groups (Figure 3a). Median percentages of CD4+CCR5+ T cells in DS and control group were $4.26(3.41 ; 5.19)$ and 4.65 (3.58; 11.3), and CD8+CCR5+ 15.10 (8.92; 18.15) and 20.65 (10.69; 35.90), respectively (Figure 3a). These differences were not statistically significant.

CD25high T regulatory cells in DS: As CD25+ cells represent both late activation marker bearing cells, as well as $T$ regulatory, suppressive cells, we next investigated the characteristics of CD25+ cells taking into account, that CD25high T cells represent typically the naturally occurring regulatory $T$ cells in humans ${ }^{16}$ (Figure 3c). Consequently, the percentage of CD25high cells was lower in DS patients than in controls in CD4+ T cell subset (Figure 3b). However, the difference in CD25high cells was not significant in CD8+ T cells.

T cell cytokine production in DS: We next performed intracellular staining to investigate major proinflammatory cytokine production in peripheral blood T cells in DS. There was no significant difference in IL-17, IFN- $\gamma$ and TNF- $\alpha$ production between healthy and DS group. Additionally, we did not see the difference in IL-4 cytokine production (Figure 4).

Monocyte characteristics in the peripheral blood of DS patients: As peripheral blood monocyte subpopulation changes might also reflect systemic chronic inflammation, we next analysed monocyte subsets based on CD14+ and CD16+ expression. We found that there were no statistically significant differences in percentage of monocytes present in the peripheral blood of both groups, as well as in percentages of three subsets of these cells: CD14highCD16-, CD14highCD16+ and CD14dimCD16+ (Figure 5). 


\section{Discussion}

Denture-related stomatitis is an inflammatory process of the oral mucosa with increasing importance in the ageing of populations, as it is one of the most common diseases among elderly denture wearers ${ }^{17,18}$. It affects around $15-70 \%$ of subjects older than 65 and is most common in complete prosthesis users ${ }^{17-19}$. The prevalence range described in the literature is wide, most probably because of different population samples investigated in different studies, but generally it appears to be more common in women ${ }^{17}$, diabetic and smoking patients ${ }^{20}$.

Systemic nature of immune activation in condition of fungal infection of the palatal tissue described as DS is still unclear. According to our hypothesis, that presence of DS in oral cavity might lead to the chronic, low grade systemic inflammation caused by persistent fungal infection. However, we did not observe in our study any relationship between presence of this condition and the percentage of lymphocytes and $T$ cells present in the peripheral blood. The same observation was also true for CD4+ and CD8+ $T$ cell subsets. Lack of differences in CD4+ $T$ cell subsets was the most surprising as this lymphocyte subset is thought to be of great importance in Candida immunity ${ }^{21}$. However, in our study group the fungal infection was connected with exogenous (denture), not an endogenous (immunosuppression caused by a viral pathogen) factor, thus the immune response may have different characteristics. Naive (CD45RA+), memory (CD45RO+) and CD28null T cells subsets were studied, because DS is frequently asymptomatic and can persist untreated for long periods of time and persistent infection provoking continuous immune activation may accelerate senescence of CD8+ T cells marked by loss of CD28 marker ${ }^{22}$. CCR5 gene expression was shown to be enhanced in peripheral blood monocytes stimulated with C. albicans in vitro ${ }^{23}$, and PBMC were shown to secrete RANTES in response to $C$. albicans presence ${ }^{24}$, therefore we studied expression of chemokine RANTES receptor CCR5 on T cells. Groups did not differ regarding to none of these cell subpopulations, probably reflecting predominantly local nature of inflammatory response in the oral mucosa in DS, which was clinically evident. We also compared amount of monocytes and their subpopulations between groups, as these cells and their differentiated form - macrophages are elements of the first line of immunologic defence against invading pathogen. Similarly, local inflammation on the oral mucosa in DS was not associated with changes of monocyte subsets. However, monocytes are very well known to play important role in inflammatory diseases such as rheumatoid arthritis or atherosclerosis ${ }^{25,26}$. It appears that especially CD16+ subsets are vital and their role in inflammatory conditions were described previously in end stage renal disease and in active rheumatoid arthritis ${ }^{25,27}$. Changes in monocyte subpopulations are visible even at very early stage of disease ${ }^{28}$, with increased amount of intermediate CD14highCD16+ and decreased amount of non-classical (CD14dimCD16+) monocytes.

In summary, lack of differences in peripheral blood immune cell subtypes may point to the lack of systemic response to oral $C$. albicans infection. While this is in contrary to our hypothesis, we believe that this is a very important consideration for interpretation of some of systemic features of DS.

While no differences were identified by our study in relation to naïve, effector or other T cell or proinflammatory monocyte subsets, we observed a lower occurrence of $T$ cells positive for late activation marker, namely CD25, in the peripheral blood of DS patients. While CD25 is classically induced by activation, it is also characteristic for naturally occurring $T$ regulatory cells ${ }^{29}$, which are characterized by a particularly high expression of CD25 (CD4+CD25high). While we have not studied 
transcription factor characteristic for Tregs - FoxP3, our preliminary experiments showed, that CD4+CD25high T cells express FoxP3 in peripheral blood in humans. Treg cells have a suppressive effect in relation to the immune system, which, under physiological conditions, is very important for the proper regulation of immune response ${ }^{29}$. In that regard, lower levels in DS patients could seem counterintuitive or indicate their exit to inflamed tissues and lower ability towards resolution of chronic inflammation, which could promote DS. Importance of T regulatory cells has, however, been recently emphasized in immune response to candidosis, during which their predominant function seems to be related to promoting pro-inflammatory Th17 type immune response, while suppressing other arms of immune response, which is vital for clearance of fungal infections ${ }^{30}$. It is also known, that patients with low number of functional Treg cells, known as IPEX (Immune Dysregulation, Polyendocrinopathy, Enteropathy, X-linked) caused by mutations in the FoxP3 gene are susceptible to infections, including Candida ${ }^{31}$.

Finally, decreased CD4+CD25high T cells could provide a link between chronic oral inflammation in DS and risk factors such as diabetes. Indeed, diabetic subjects were overrepresented in DS study group, and presence of this condition is associated with lower numbers of CD25+ T cells ${ }^{32}$. These results may suggest that lower $C D 4+C D 25+T$ cells in DS could link mechanistically these two conditions, although subsequent studies characterizing these mechanisms are warranted. Thus, unexpectedly, our results may point our attention towards a possible mechanism of chronic inflammation in DS patients rather, than showing systemic consequences of this process.

Our study has some limitations - all of the experiments were performed in a relatively small number of patients and, as such, should be considered preliminary, although up to date no other study has systematically addressed the issue of peripheral T cell and monocyte activation in relation to DS. It should be also kept in mind, that presence of confounders, such as diabetes mellitus or smoking, although not statistically significant, can affect the results. While studied group is insufficient to control for these factors statistically, we should bear in mind that these are genuine and strong risk factors of the disease, so lack of difference between groups would render this comparison clinically less meaningful. The caution is also needed also to make wide conclusion on general population, as predominance of women in both study groups was obvious, although again linked to DS epidemiology. Finally, while cytometric methods used in the present study are considered sensitive and accurate, their sensitivity may not be sufficient to detect extremely subtle differences in markers and mediators ${ }^{33}$. However, such differences would be unlikely to have clinical relevance.

In summary, although immune mechanisms involved in the local immune defence against oral fungal infection are very complex and include various cell types - not only immune cells $s^{4,21,34}$, but also epithelial cells of the infected tissues ${ }^{35}$, the main observation of our work is the lack of the evidence of systemic cellular response to oral $C$. albicans infection. At the same time, differences regarding CD4+CD25high+ T cell subsets may point to the potential role of Treg cells in susceptibility of diabetic patients to denture-related stomatitis. Knowledge of the detailed mechanism responsible for increasing DS risk in diabetics may allow for an effective prevention of oral fungal infections in this population, thus improving comfort of denture usage and patient's quality of life. Prevention of DS might also be of particular importance, in the light of our previous work, where higher prevalence of endothelial dysfunction in DS patients was observed ${ }^{1}$. Endothelial dysfunction as a symptom preceding the development of atherosclerosis ${ }^{36}$, may have serious consequences for the health and life of the patients. Therefore, reducing the risk of its occurrence constitutes an important clinical 
goal. Growing body of evidence suggests, that Th17 immune response, plays an important role in immune responses to mucosal and cutaneous candidosis ${ }^{37}$. Recently discovered role of these proinflammatory mechanisms in hypertension ${ }^{38,39}$ makes these immune cells a particularly interesting target for research looking into the mechanisms linking oral and cardiovascular disease.

\section{Acknowledgments}

This study was supported by the Foundation for Polish Science Welcome Grant (FNP/Welcome/02) (TJG, TM, GO, AS) and public Funds for Science of Republic of Poland grant Nr 2997/B/P01/2009/36 and

\section{Conflict of interests}

The authors declare that there is no conflict of interests regarding the publication of this paper. 


\section{References}

1. Maciąg J, Osmenda G, Nowakowski D, et al.: Denture-related stomatitis is associated with endothelial dysfunction. BioMed Res Int 2014;2014:474016

2. Hajishengallis G: Immunomicrobial pathogenesis of periodontitis: keystones, pathobionts, and host response. Trends Immunol 2014;35:3-11

3. Martori E, Ayuso-Montero R, Martinez-Gomis J, et al.: Risk factors for denture-related oral mucosal lesions in a geriatric population. J Prosthet Dent 2014;111:273-9

4. Schaller M, Boeld U, Oberbauer S, et al.: Polymorphonuclear leukocytes (PMNs) induce protective Th1-type cytokine epithelial responses in an in vitro model of oral candidosis. Microbiol Read Engl 2004;150:2807-13

5. Gasparoto TH, Vieira NA, Porto VC, et al.: Ageing exacerbates damage of systemic and salivary neutrophils from patients presenting Candida-related denture stomatitis. Immun Ageing A 2009;6:3

6. Naglik JR, Moyes DL, Wächtler B, et al.: Candida albicans interactions with epithelial cells and mucosal immunity. Microbes Infect Inst Pasteur 2011;13:963-76

7. Gaffen SL, Herzberg MC, Taubman MA, et al.: Recent advances in host defense mechanisms/therapies against oral infectious diseases and consequences for systemic disease. Adv Dent Res 2014;26:30-7

8. Conti HR, Shen F, Nayyar N, et al.: Th17 cells and IL-17 receptor signaling are essential for mucosal host defense against oral candidiasis. J Exp Med 2009;206:299-311

9. Milner JD, Brenchley JM, Laurence $A$, et al.: Impaired $\mathrm{T}(\mathrm{H}) 17$ cell differentiation in subjects with autosomal dominant hyper-IgE syndrome. Nature 2008;452:773-6

10. Kisand K, Bøe Wolff AS, Podkrajsek KT, et al.: Chronic mucocutaneous candidiasis in APECED or thymoma patients correlates with autoimmunity to Th17-associated cytokines. J Exp Med 2010;207:299-308

11. Puel A, Cypowyj S, Bustamante J, et al.: Chronic mucocutaneous candidiasis in humans with inborn errors of interleukin-17 immunity. Science 2011;332:65-8

12. van de Veerdonk FL, Plantinga TS, Hoischen A, et al.: STAT1 mutations in autosomal dominant chronic mucocutaneous candidiasis. N Engl J Med 2011;365:54-61

13. Oliveira MAM de, Carvalho LP, Gomes M de S, et al.: Microbiological and immunological features of oral candidiasis. Microbiol Immunol 2007;51:713-9

14. Rodriguez-Archilla A, Urquia M, Cutando A, et al.: Denture stomatitis: quantification of interleukin-2 production by mononuclear blood cells cultured with Candida albicans. J Prosthet Dent 1996;75:426-31 
15. Perk J, De Backer G, Gohlke H, et al.: European Guidelines on cardiovascular disease prevention in clinical practice (version 2012): The Fifth Joint Task Force of the European Society of Cardiology and Other Societies on Cardiovascular Disease Prevention in Clinical Practice (constituted by representatives of nine societies and by invited experts). Atherosclerosis 2012;223:1-68

16. van der Geest KSM, Abdulahad WH, Tete SM, et al.: Aging disturbs the balance between effector and regulatory CD4+ T cells. Exp Gerontol 2014;60:190-6

17. Gendreau L, Loewy ZG: Epidemiology and etiology of denture stomatitis. J Prosthodont Off J Am Coll Prosthodont 2011;20:251-60

18. Kossioni AE: The prevalence of denture stomatitis and its predisposing conditions in an older Greek population. Gerodontology 2011;28:85-90

19. Abaci O, Haliki-Uztan A, Ozturk B, et al.: Determining Candida spp. incidence in denture wearers. Mycopathologia 2010;169:365-72

20. Dorocka-Bobkowska B, Zozulinska-Ziolkiewicz D, Wierusz-Wysocka B, et al.: Candidaassociated denture stomatitis in type 2 diabetes mellitus. Diabetes Res Clin Pract 2010;90:81-6

21. Villar CC, Dongari-Bagtzoglou A: Immune defence mechanisms and immunoenhancement strategies in oropharyngeal candidiasis. Expert Rev Mol Med 2008;10:e29

22. Vallejo AN, Weyand CM, Goronzy JJ: T-cell senescence: a culprit of immune abnormalities in chronic inflammation and persistent infection. Trends Mol Med 2004;10:119-24

23. Kim HS, Choi EH, Khan J, et al.: Expression of genes encoding innate host defense molecules in normal human monocytes in response to Candida albicans. Infect Immun 2005;73:3714-24

24. Huang C, Levitz SM: Stimulation of macrophage inflammatory protein-1alpha, macrophage inflammatory protein-1beta, and RANTES by Candida albicans and Cryptococcus neoformans in peripheral blood mononuclear cells from persons with and without human immunodeficiency virus infection. J Infect Dis 2000;181:791-4

25. Kinne RW, Bräuer R, Stuhlmüller B, et al.: Macrophages in rheumatoid arthritis. Arthritis Res 2000;2:189-202

26. Hansson GK: Inflammation, atherosclerosis, and coronary artery disease. N Engl J Med 2005;352:1685-95

27. Kawanaka N, Yamamura M, Aita T, et al.: CD14+,CD16+ blood monocytes and joint inflammation in rheumatoid arthritis. Arthritis Rheum 2002;46:2578-86

28. Klimek E, Mikołajczyk T, Sulicka J, et al.: Blood monocyte subsets and selected cardiovascular risk markers in rheumatoid arthritis of short duration in relation to disease activity. BioMed Res Int 2014;2014:736853

29. Sakaguchi S, Wing K, Miyara M: Regulatory T cells - a brief history and perspective. Eur J Immunol 2007;37 Suppl 1:S116-23 
30. Pandiyan P, Conti HR, Zheng L, et al.: CD4+ CD25+ Foxp3+ regulatory T cells promote Th17 cells in vitro and enhance host resistance in mouse Candida albicans Th17 cell infection model. Immunity 2011;34:422-34

31. Ochs HD, Gambineri E, Torgerson TR: IPEX, FOXP3 and regulatory T-cells: a model for autoimmunity. Immunol Res 2007;38:112-21

32. Zeng C, Shi X, Zhang B, et al.: The imbalance of Th17/Th1/Tregs in patients with type 2 diabetes: relationship with metabolic factors and complications. J Mol Med Berl Ger 2012;90:175-86

33. Gongora MC, Lob HE, Landmesser $\mathrm{U}$, et al.: Loss of extracellular superoxide dismutase leads to acute lung damage in the presence of ambient air: a potential mechanism underlying adult respiratory distress syndrome. Am J Pathol 2008;173:915-26

34. Upadhyay J, Upadhyay RB, Agrawal P, et al.: Langerhans Cells and Their Role in Oral Mucosal Diseases. North Am J Med Sci 2013;5:505-14

35. Naglik JR, Moyes D: Epithelial cell innate response to Candida albicans. Adv Dent Res 2011;23:50-5

36. Guzik TJ, West NE, Black E, et al.: Vascular superoxide production by NAD(P)H oxidase: association with endothelial dysfunction and clinical risk factors. Circ Res 2000;86:E85-90

37. Filler SG: Insights from human studies into the host defense against candidiasis. Cytokine 2012;58:129-32

38. Harrison DG, Guzik TJ, Lob HE, et al.: Inflammation, immunity, and hypertension. Hypertension 2011;57:132-40

39. Guzik TJ, Hoch NE, Brown KA, et al.: Role of the T cell in the genesis of angiotensin II induced hypertension and vascular dysfunction. J Exp Med 2007;204:2449-60 
Table 1. Studied groups clinical characteristics. Data are presented as mean (SD) or $n(\%)$.

\begin{tabular}{lccc}
\hline & DS patients & Control group & P value \\
\hline Number of patients & 20 & 24 & \\
Age [years] & $63.9(6.6)$ & $65.9(10.3)$ & 0.425 \\
BMI & $29.7(5.8)$ & $27.9(5.3)$ & 0.297 \\
Gender (M:F) & $2: 18$ & $6: 18$ & 0.259 \\
Partial upper denture & $5(25 \%)$ & $3(12.5 \%)$ & 0.436 \\
Complete upper dentures & $15(75 \%)$ & $21(87.5 \%)$ & 0.436 \\
Current smoking & $6(30 \%)$ & $3(12.5 \%)$ & 0.261 \\
Smoking in the past & $2(10 \%)$ & $10(41.7 \%)$ & 0.083 \\
Diabetes & $6(30 \%)$ & $2(8.3 \%)$ & 0.115 \\
Coronary artery disease & $9(45 \%)$ & $8(33.3 \%)$ & 0.631 \\
Controlled hypertension & $8(40 \%)$ & $10(41.7 \%)$ & 0.845 \\
Untreated hypertension & $9(45 \%)$ & $10(41.7 \%)$ & 0.934 \\
\hline
\end{tabular}

BMI - body mass index; DS - denture-related stomatitis; F - females; M - males; SD - standard deviation 
Figure 1. Maciąg et al
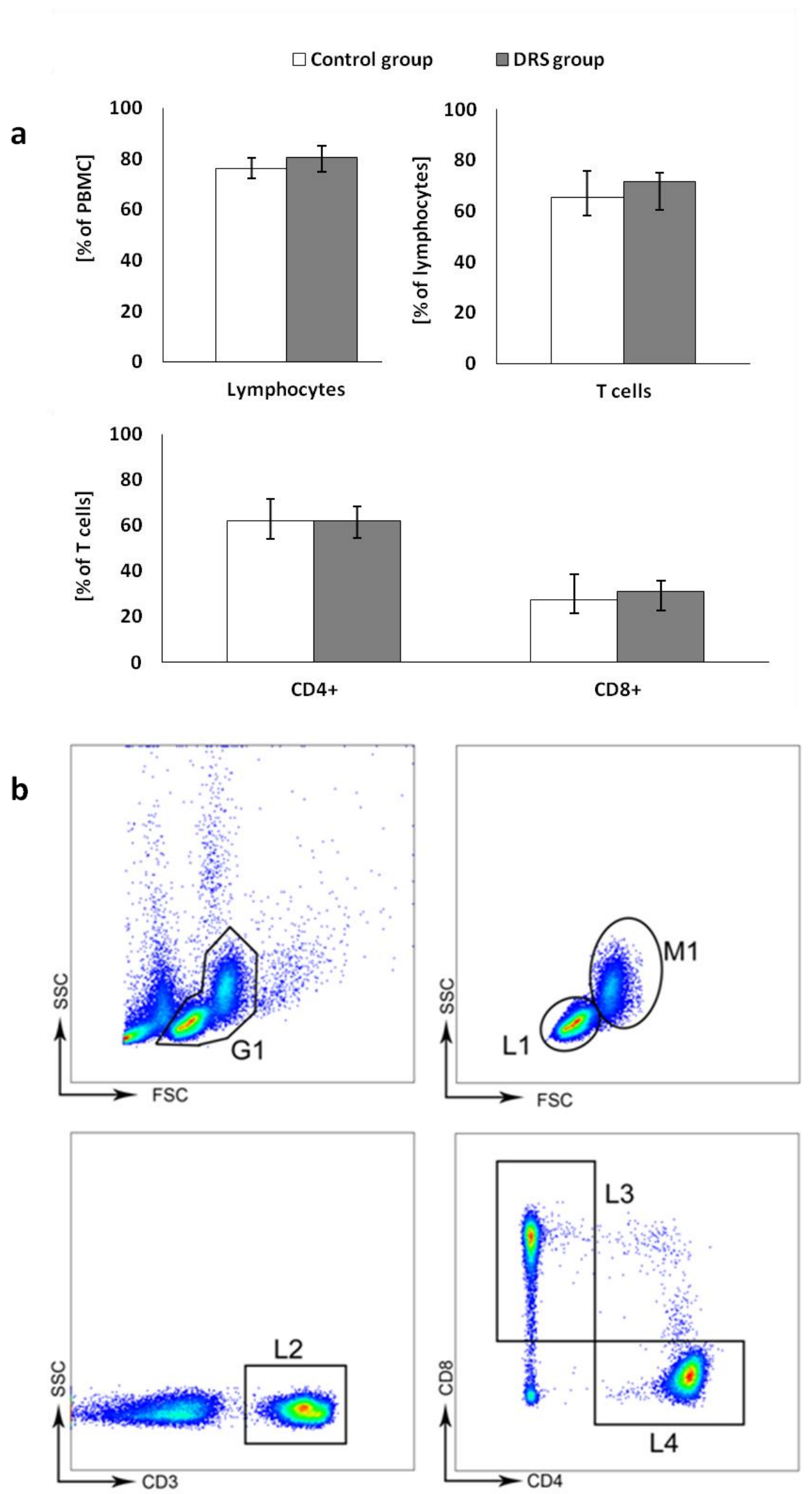
Figure 2. Maciąg et al

a

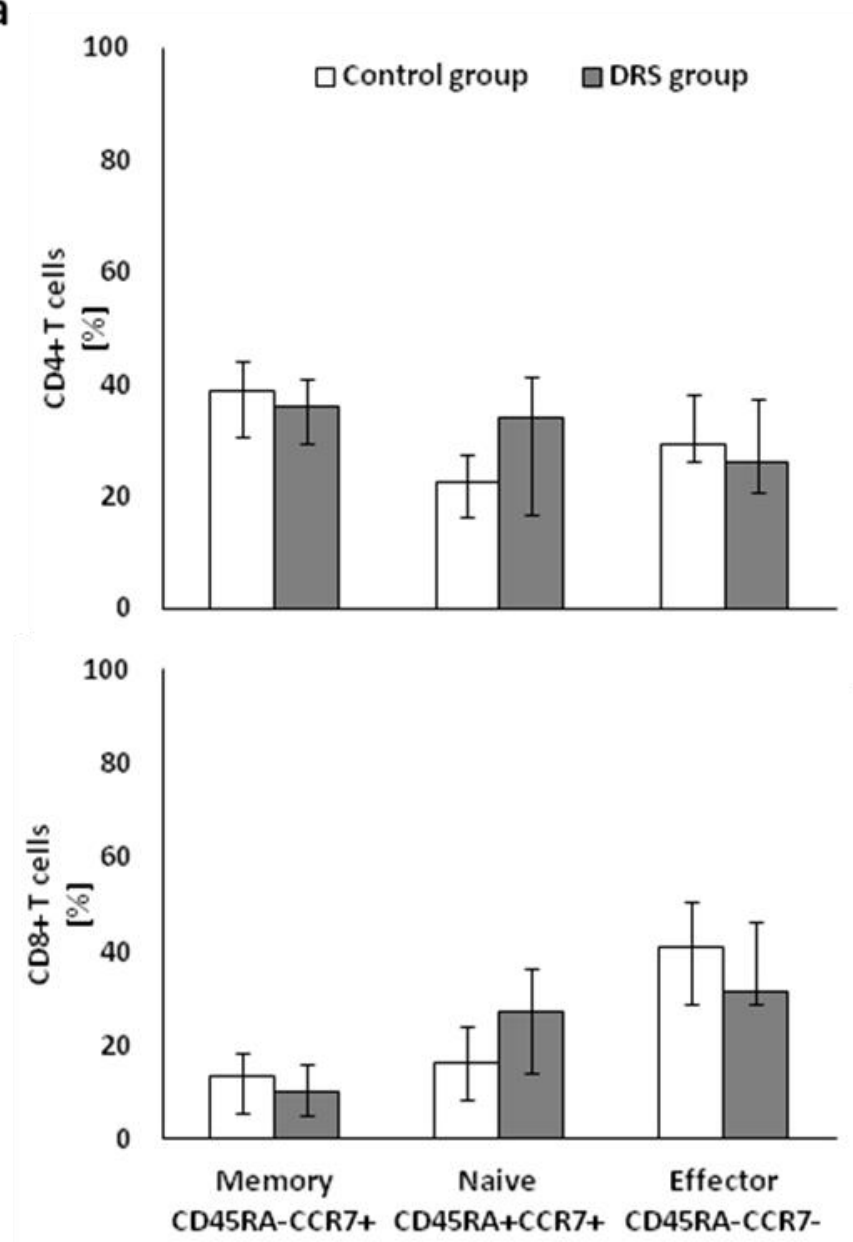

b
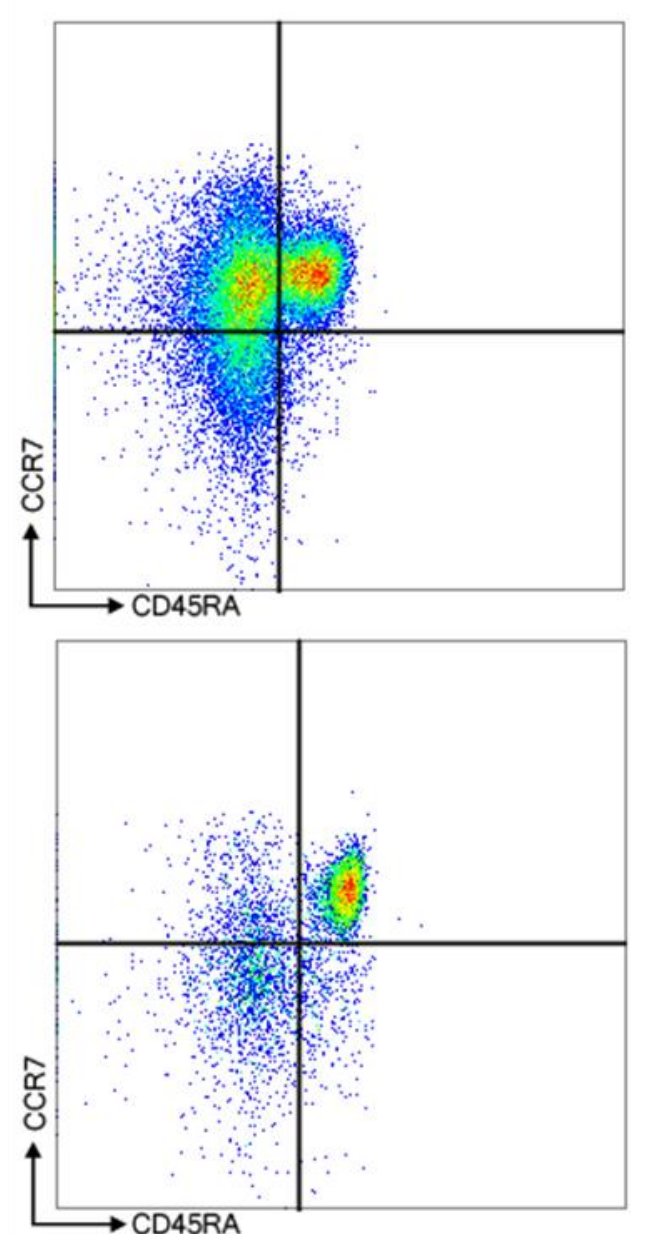
Figure 3. Maciąg et al

a
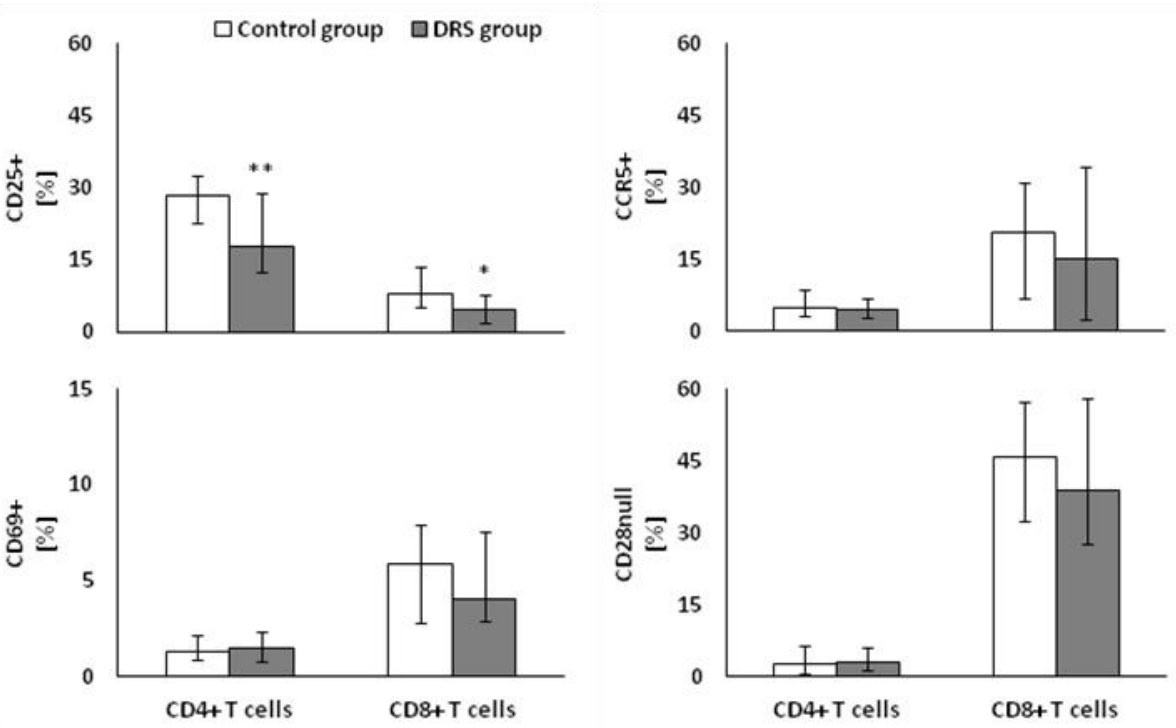

b

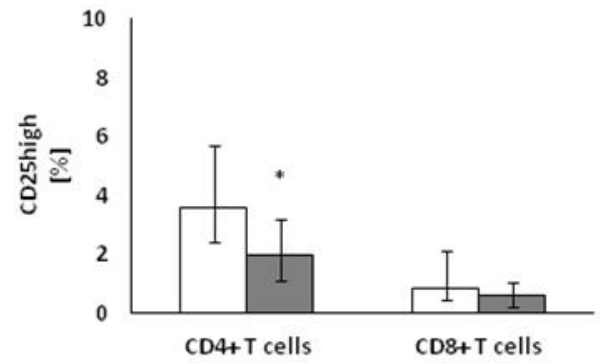

c
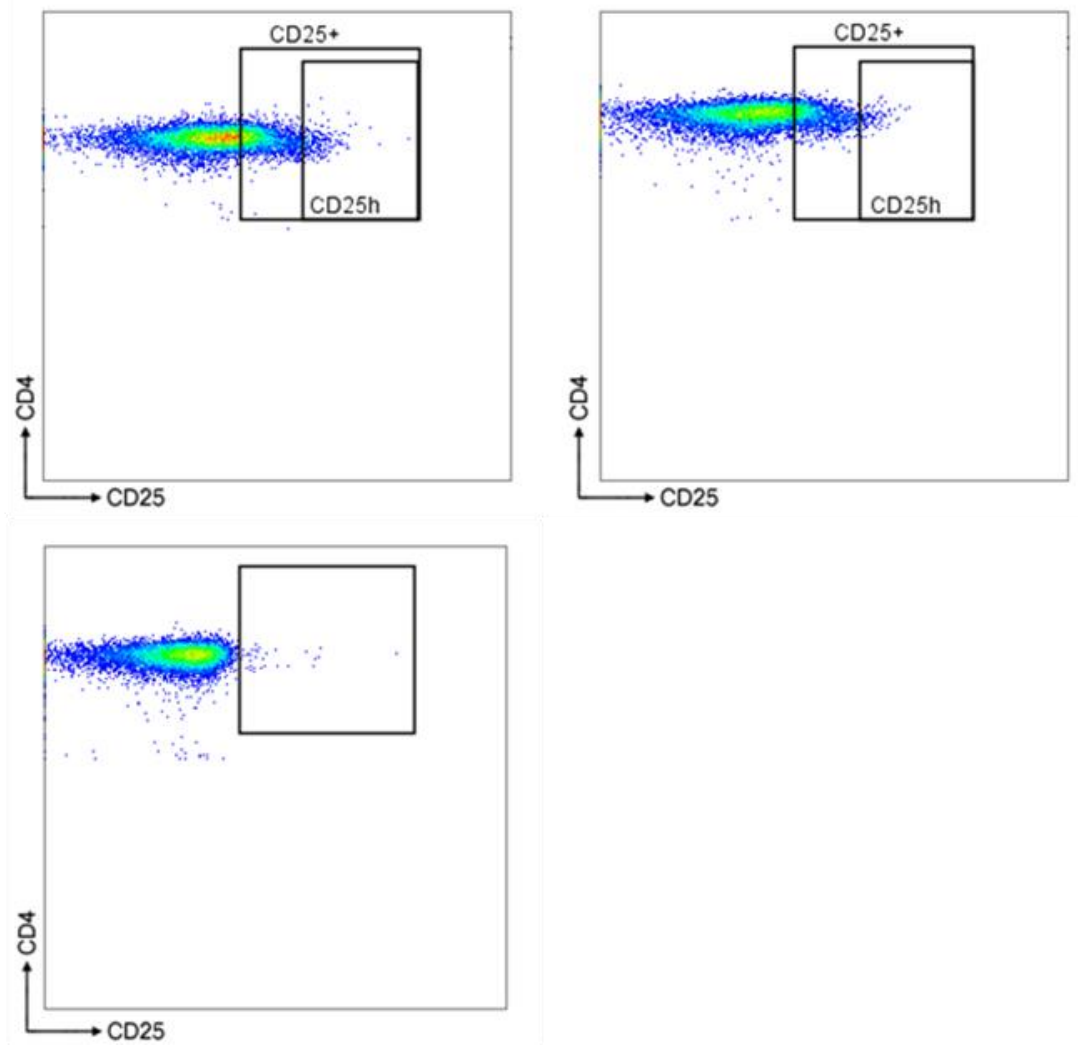
Figure 4. Maciąg et al

a
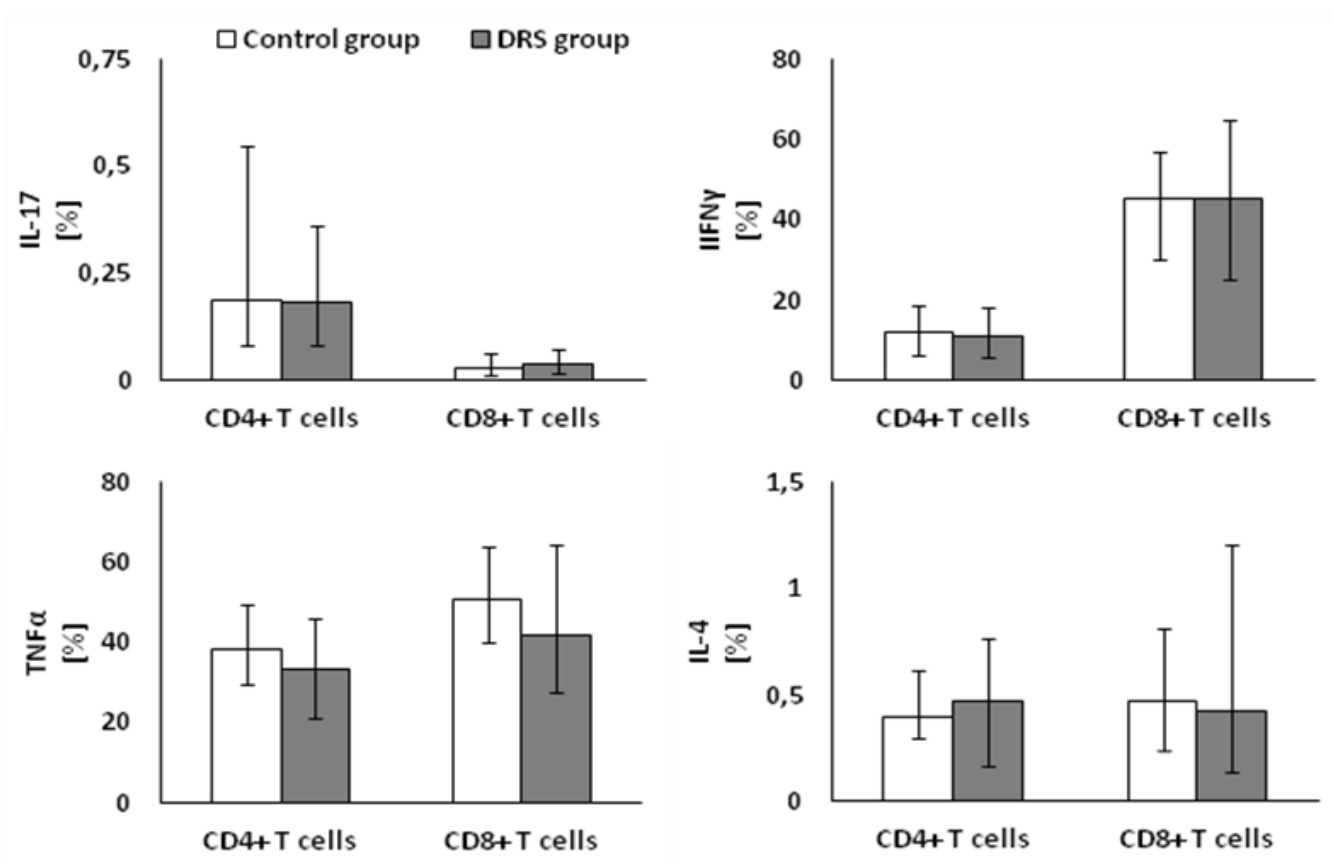

b

CD4 T cells

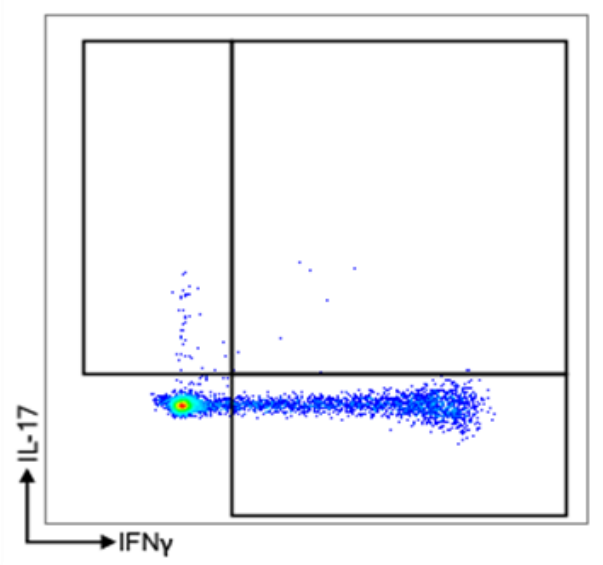

CD8 T cells
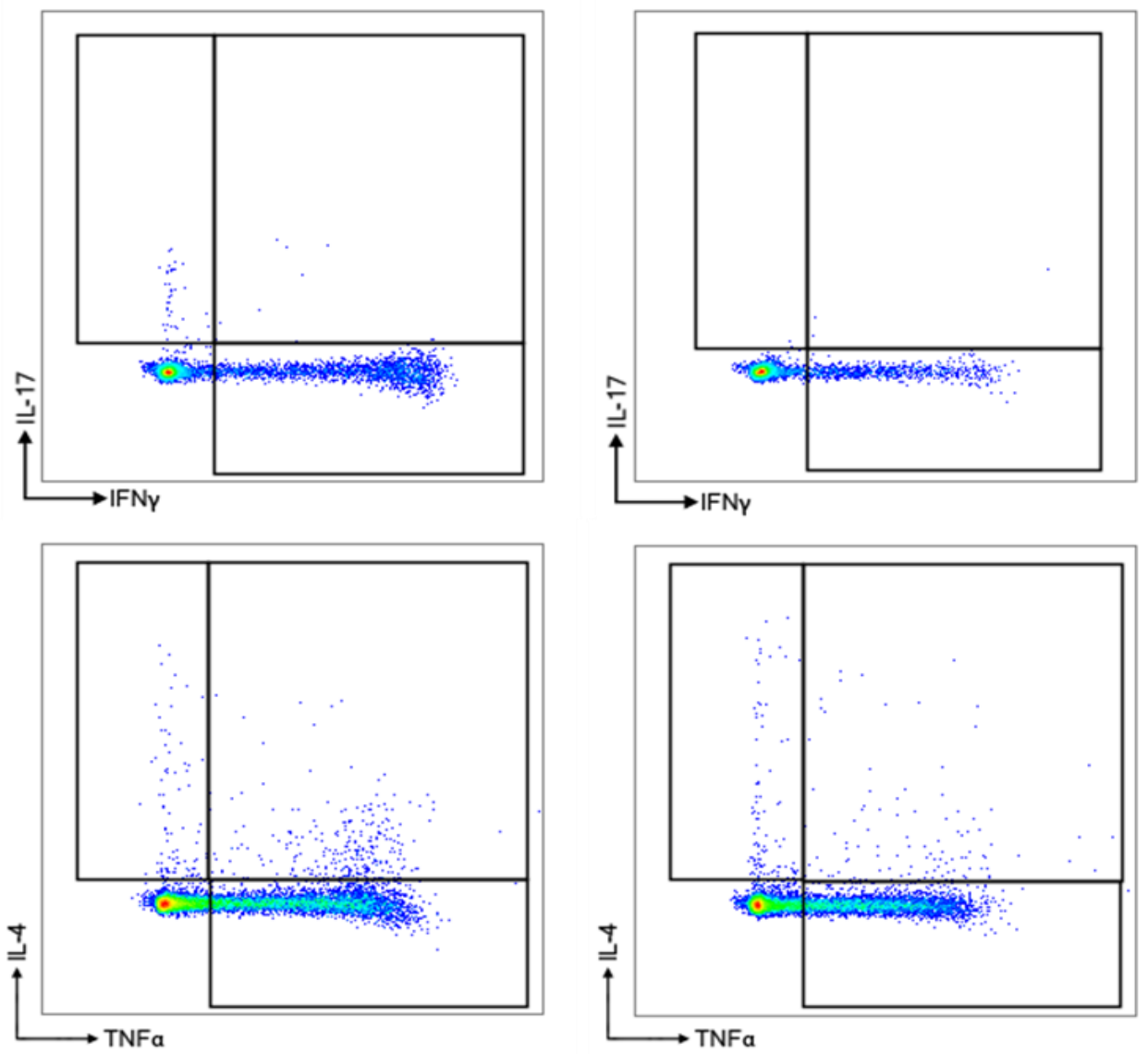
Figure 5. Maciąg et al

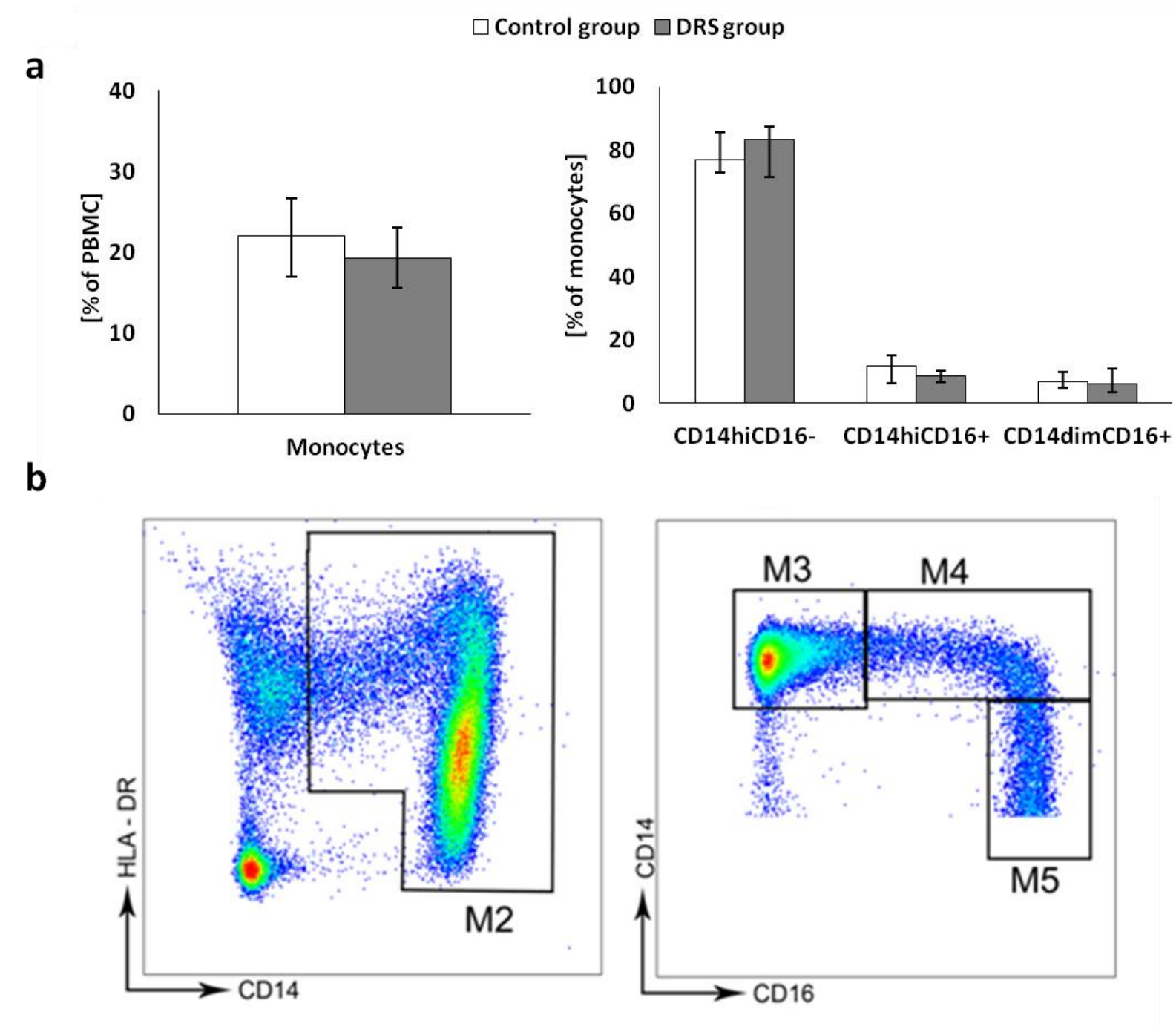


Figure legends:

Figure 1. (a) Lymphocytes and their basic subpopulations in DS and control patients. Comparison of lymphocytes and T cells counts and basic T cell subpopulations: CD4+ and CD8+ in control and DS patients. Lymphocyte subpopulation was measured in peripheral blood by flow cytometry. Results presented as median (Q1; Q2). (b) Example of flow cytometric gating strategy in PBMC. Gating of lymphocytes and their subsets: G1 - PBMC, L1 - lymphocytes, M1 - monocytes, L2 - T cells, L3 - CD8+ T cells, L4 - CD4+ T cells.

Figure 2. (a) Subsets of the naive, effector and memory CD4+ and CD8+ T cells present in peripheral blood of DS and control patients. Counts of the naive (CD45RA+CCR7+), effector (CD45RA-CCR7-) and memory (CD45RA-CCR7+) T cells were assessed by flow cytometry. Results presented as median (Q1; Q2). (b) Example of flow cytometric results and gating strategy for naive, effector and memory CD4+ (upper panel) and CD8+ (lower panel) subsets.

Figure 3. (a) Comparison of percentage of activated T cells and CCR5+ and of CD28null T cells of DS and control patients in CD4 and CD8 subset of lymphocytes. Expression of late activation marker (CD25), early activation marker (CD69) and chemokine RANTES receptor CCR5 in CD4 and CD8 subsets and prevalence of CD4+ CD28null and CD8+CD28null cells were studied in peripheral blood by flow cytometry. Data are presented as \% of cells expressing individual marker. Results presented as median (Q1; Q2). * - $p=0,0076 ;{ }^{* *}-p=0,0006$. (b) Presence of CD25high (h) T cells in DS and control patients. Results presented as median (Q1; Q2). * $-p=0,0012$. (c) Example of flow cytometric results for CD25high CD4+ T cell subsets for DS (upper right) and control (upper left) groups. CD25+ gate was placed on the basis of isotype control staining (lower left).

Figure 4. (a) Results of intracellular staining of CD4+ and CD8+ T cell subsets in peripheral blood of DS and control patients. Percentage of cells producing IL-4, IL-17, TNF $\alpha$ or IFN $\gamma$ was assessed by flow cytometry. Results presented as median (Q1; Q2). (b) Example of flow cytometric results and gating strategy of intracellular staining for CD4+ (left) and CD8+ (right) T cell subsets.

Figure 5. (a) Comparison of monocytes and monocyte subsets in peripheral blood of DS and control patients. Counts of the presented subpopulations were assessed by flow cytometry. Results presented as median (Q1; Q2); $\mathrm{n}$ control group $=24, \mathrm{n} D S=19$. (b) Example of flow cytometric gating strategy of monocytes and their subpopulations. Gating of monocytes and their subsets: M2 gate applied to exclude CD14-HLA-DR- cells, M3 - CD14highCD16-, M4 - CD14highCD16+, M5 CD14dimCD16+. 